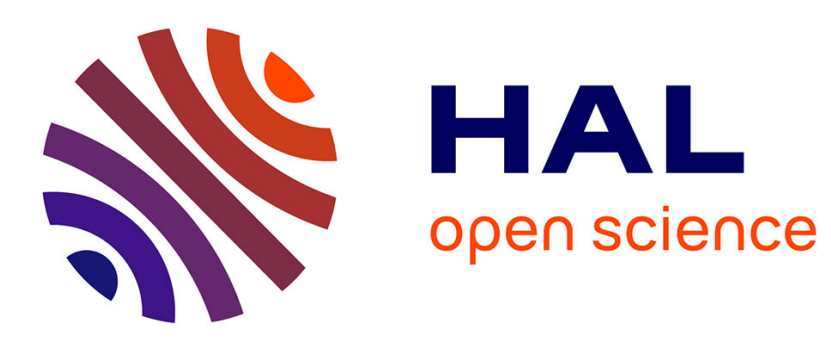

\title{
EXPERIMENTAL EVIDENCES FOR AN HALDANE GAP IN QUASI ONE-DIMENSIONAL ANTIFERROMAGNETS
}

J. Renard, L. Regnault, M. Verdaguer

\section{- To cite this version:}

J. Renard, L. Regnault, M. Verdaguer. EXPERIMENTAL EVIDENCES FOR AN HALDANE GAP IN QUASI ONE-DIMENSIONAL ANTIFERROMAGNETS. Journal de Physique Colloques, 1988, 49 (C8), pp.C8-1425-C8-1429. 10.1051/jphyscol:19888655 . jpa-00228885

\section{HAL Id: jpa-00228885 https://hal.science/jpa-00228885}

Submitted on 1 Jan 1988

HAL is a multi-disciplinary open access archive for the deposit and dissemination of scientific research documents, whether they are published or not. The documents may come from teaching and research institutions in France or abroad, or from public or private research centers.
L'archive ouverte pluridisciplinaire HAL, est destinée au dépôt et à la diffusion de documents scientifiques de niveau recherche, publiés ou non, émanant des établissements d'enseignement et de recherche français ou étrangers, des laboratoires publics ou privés. 


\title{
EXPERIMENTAL EVIDENCES FOR AN HALDANE GAP IN QUASI ONE-DIMENSIONAL ANTIFERROMAGNETS
}

\author{
J. P. Renard $\left({ }^{1}\right)$, L. P. Regnault $\left({ }^{2}\right)$ and M. Verdaguer $\left({ }^{3}\right)$ \\ (1) Institut d'Electronique Fondamentale, CNRS UA 022, Bât. 220 Université Paris Sud, F-91405 Orsay Cedex, \\ France \\ $\left({ }^{2}\right)$ Centre d'Etudes Nucléaires, DRF/SPh-MDN, 85X, 38041 Grenoble Cedex, France \\ $\left({ }^{3}\right)$ Laboratoire de Spectrochimie des Elêments de Transition, CNRS UA420, Université Paris*Sud, 91405, Orsay, \\ France
}

\begin{abstract}
It has been predicted by Haldane that the one-dimensional (1D) Heisenberg antiferromagnet (AF) with integer spin has a singlet ground state separated from the excited states by an energy gap. The striking susceptibility decrease as $T \rightarrow 0$ and the absence of long range order in quasi- $1 \mathrm{D}$-AF compounds with $S=1, \mathrm{Ni}_{(}\left(\mathrm{C}_{2} \mathrm{H}_{8} \mathrm{~N}_{2}\right)_{2} \mathrm{NO}_{2} \mathrm{ClO}_{4}$ and two other related $\mathrm{Ni}$ chains, and in $\mathrm{AgVP}_{2} \mathrm{~S}_{6}$ support the existence of Haldane gap. More detailed experiments including inelastic neutron scatering in NENP reveal a splitting of the Haldane gap by planar anisotropy, in agreement with numerical calculations.
\end{abstract}

\section{Introduction}

One-dimensional (1D) magnetic systems are of large current interest for the peculiar quantum effects they exhibit at low temperature [1] and the possibilities of mapping $1 \mathrm{D}$ spin models to $2 \mathrm{D}$ interacting fermions $[2]$.

A striking phenomenon is the strongly spin dependent behavior at $T=0$, of the 1D Heisenberg antiferromagnet (1D-HAF). As assumed by Haldane [1], for integer values of the spin $S$ the 1D-HAF exhibits a singlet ground state separated from the first triplet excited state by an energy gap $E_{\mathbf{G}}$, while for half-odd integer $S$ the $1 D-H A F$ has a gapless spectrum of excitations. The spin correlation function is also completely different for integer and half-odd integer $S$. In chains where $S$ is integer, the pair spin correlation $\left\langle S_{0} S_{n}\right\rangle$ has an exponential decay with the distance $n$ and thus the correlation length is limited to a finite value $\xi_{0}$ as $T$ tends to zero. This differs strongly to the halfodd integer case where $\left\langle S_{0} S_{n}\right\rangle$ has the power law decay $(-1)^{n}|n|^{-1}[1,3]$ leading to an infinite correlation length at $T=0$.

Up to now, there is no simple physical picture of the Haldane gap. Recently, Affleck et al. [4] have obtained exact valence bond ground states of an extended Heisenberg model containing additional terms of higher order than bilinear. Their treatment which confirms the Haldane's conjecture gives for $S=1$, a correlation length of about the distance between nearest neighbor spins $\xi_{0}=1 / \operatorname{Ln} 3$.

The numerical calculations on finite rings of exchange coupled spins $S=1$ [5-9], also well support the Haldane's conjecture. Monte-Carlo calculations up to 32 spins [10] provide a reliable value of the energy gap $E_{\mathrm{G}}=0.41|\mathrm{~J}|$ for the $1 \mathrm{D}-\mathrm{HAF}$ with nearest neighbor exchange interaction $-J \mathbf{S}_{i} . \mathbf{S}_{i+1}$. The effect of exchange anisotropy. ( $X X Z$ model), and single ion anisotropy $D\left(S_{i}^{z}\right)^{2}$ has been investigated by different numerical techniques. A very important result for a practical point of view, is the persistence of the gap for not too large anisotropy values. This is exemplified in the figure 1 which shows the energy gap of the 1D-HAF with single ion anisotropy described by the following hamiltonian:

$$
\mathcal{H}=\sum_{i}\left[-J\left(S_{i}, S_{i+1}\right)+D\left(S_{i}^{z}\right)^{2}\right]
$$

\section{Conditions for observation of Haldane gap}

In the ideal Heisenberg chain of integer spins, the low temperature magnetic properties are strongly affected by the presence of a gap in the magnetic excitations. At temperatures below $E_{\mathrm{G}} / k$, the magnetic susceptibility is expected to decrease rapidly and to tend to a value close to zero as $T \rightarrow 0$, independently of the magnetic field orientation. The specific heat will depart from the $T$ law, predicted from the spinwave theory and established by Takahashi [11] for the $S=1 / 2,1 \mathrm{D}$-HAF .

In quasi 1D-HAF with integer spin, there is a competition betwen the gap which tends to maintain a non-ordered phase at low $T$ and the interchain interaction $J^{\prime}$ which tends to induce 3D long range order (LRO). Kosevich et al. [12] have shown that for $\left|J^{\prime} / J\right|$ below a critical value which scales like $\exp (-\pi S), 3 \mathrm{D}$ LRO cannot be achieved at non zero temperature. The observation of the Haldane gap requires spin 1, small ratio of interchain to intrachain exchange interaction, not too large magnetic anisotropy (see Fig. 1) and no exchange alternation. Due to the quantum origin of the gap, its size is a rapidly decreasing function of spin, as $|J| S^{2} \exp (-\pi S)$ for large $S$. So there is little chance for observing the gap in chains with $S=2$. 


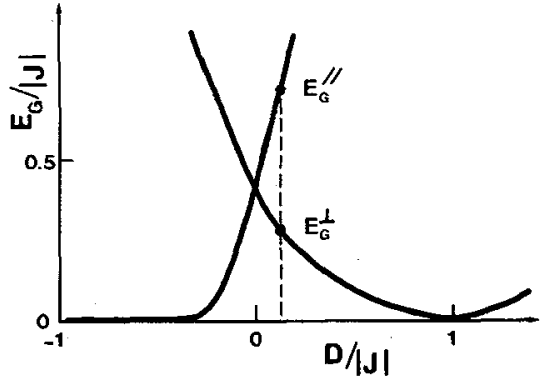

Fig. 1. - Sketch of the variation of the Haldane gap with single ion anisotropy $D$. The dashed line corresponds to the case of small planar anisotropy encountered in NENP.

\section{Experiments on quasi-1D antiferromagnets with $\mathbf{S}=\mathbf{1}$}

3.1 INTRODUCTION. - The first attempts for observing the gap in quasi-1D, HAF were done by neutron scattering experiments in $\mathrm{Cs} \mathrm{Ni} \mathrm{Cl}_{3}[13,14]$. this compound has the disadvantage to have $3 \mathrm{D}$-LRO below $T_{\mathrm{N}}=4.85 \mathrm{~K}$. This restricts the useful temperature range for the gap observation to $k T \simeq E_{\mathrm{G}}$ where thermal broadening is important. In $\mathrm{Cs} \mathrm{Ni} \mathrm{Cl}$ the $\mathrm{Ni}^{2+}$ chains are too close from each other leading to relatively large $\left|J^{\prime} / J\right|$ value of about $10^{-2}$. A way for reducing $\left|J^{\prime} / J\right|$ consists in increasing the chain spacing while keeping a large $|J|$ value. this is realized in $\mathrm{Ni}\left(\mathrm{N}_{2} \mathrm{C}_{2} \mathrm{H}_{8}\right)_{2} \mathrm{NO}_{2} \mathrm{ClO}_{4}$, alias NENP, an orthorhombic crystal of $\mathrm{Ni}^{2+}$ chains well isolated from each other by $\mathrm{ClO}_{4}^{-}$ions [15] (Fig. 2). We succeeded to grow large single crystals of NENP allowing several experiments by different techniques which well supported the Haldane's conjecture $[16,17]$. Other $\mathrm{Ni}^{2+}$ chain compounds structurally related to NENP are also good candidates for Haldane gap studies (Tab. I). An other $S=1$ chain compound $\mathrm{AgVP}_{2} \mathrm{~S}_{6}$. recently studied by P. Colombet et al. [18] is very attractive because of its large intrachain interaction and its relatively simple structure. This later one derives from the lamellar structure of $\mathrm{MPS}_{3}$ by substitution of the $2 \mathrm{M}^{2+}$ ions by the couple of $\mathrm{Ag}^{+}+\mathrm{V}^{3+}$. The $\mathrm{Ag}^{+}$and $\mathrm{V}^{3+}$ ions form zigzag chains shown in figure 2. Finally it should

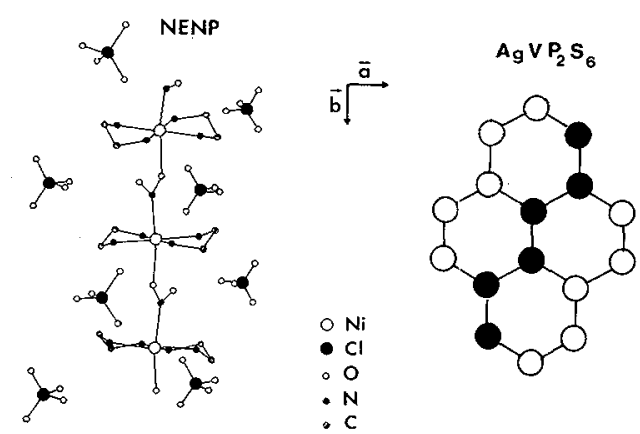

Fig. 2. - Schematic view of the chain structure of $\mathrm{Ni}\left(\mathrm{C}_{2} \mathrm{H}_{8} \mathrm{~N}_{2}\right)_{2} \mathrm{NO}_{2} \mathrm{ClO}_{4}(\mathrm{NENP})$ and $\mathrm{AgVP}_{2} \mathrm{~S}_{6}$.

be mentionned that chains of $S=1 / 2$ ferromagnetic dimers weakly coupled by AF interaction [19] could mimic the $S=1$, ID-HAF at low temperature. The main magnetic characteristics of the preceeding chain compounds are listed in table I.

3.2 SUSCEPTIBILITY DATA. - The drastic decrease of the magnetic susceptibility $\chi(T)$ as $T \rightarrow 0$, expected for the $S=1,1 \mathrm{D}-\mathrm{AF}$ with a gap, is well evidenced by the experimental data in single crystals of NENP (Fig. 3). In the gapless $1 \mathrm{D}-\mathrm{HAF}$, the ratio $\chi(0) / \chi_{\max }$ of the susceptibility at $T=0$ to its maximum value is slightly smaller than 1: $\chi(0) / \chi_{\max }=0.69$ for $S=1 / 2$ and 0.83 for $S=\infty$. In NENP, this ratio is found below 0.1 for all field orientations. Along the hard axis $b$, the tendency to low temperature $\chi^{l l}$ decrease is less marked than along the easy axes of $a c$ plane, as expected for an AF chain. 'Thus the effect of the gap on $\chi \|$ occurs at lower temperatures than for $\chi^{\perp}$. A rough estimate of the gap was obtained by fitting the low $T \chi^{\perp}$ data to an exponential decrease [16]: $E_{\mathrm{G}}^{\perp} / 17 \mathrm{~K}$. This can be compared to the temperature $T_{1 / 2}$ for which $\chi^{\perp}(T)=\chi_{\max }^{\perp} / 2: T_{1 / 2} \simeq 13 \mathrm{~K}$.

The effect of $\mathrm{Cu}^{2+}$ impurity substituted to $\mathrm{Ni}^{2+}$ at low atomic concentration $x$ on $\chi^{\|}$and $\chi^{\perp}$ has been investigated on single crystals of NENJP. The susceptibility curves for $x=3.6 \times 10^{-3}$ and $6.2 \times 10^{-3}$ show a large impurity contribution to $\chi(T)$ (Fig. 4). For $\chi^{\perp}$ (easy plane), but not for $\chi \|$, the impurity contribution

Table I. - Main characteristics of some $S=1$ chain compounds. $\mathrm{NENP}=\mathrm{Ni}\left(\mathrm{C}_{2} \mathrm{H}_{8} \mathrm{~N}_{2}\right)_{2} \mathrm{NO}_{2} \mathrm{ClO}_{4} ; \mathrm{NINO}=$ $\mathrm{Ni}\left(\mathrm{C}_{3} \mathrm{H}_{10} \mathrm{~N}_{2}\right)_{2} \mathrm{NO}_{2} \mathrm{ClO}_{4} ; \mathrm{NINAZ}=\mathrm{Ni}\left(\mathrm{C}_{3} \mathrm{H}_{10} \mathrm{~N}_{2}\right)_{2} \mathrm{~N}_{3} \mathrm{ClO}_{4} ; \mathrm{CuCl}_{3}-\mathrm{BP}=\mathrm{CuCl}_{3}$ (4-benzyl-piperidinium).

$\begin{array}{cccccc}\text { Compound } & J / k(\mathrm{~K}) & \left|J^{\prime} / J\right| & D /|J| & T_{\mathrm{N}}(\mathrm{K}) & \text { Ref. } \\ & & & & & \\ \mathrm{CsNiCl}_{3} & -30 & 1.7 \times 10^{-2} & -0.02 & 4.85 & {[13,14]} \\ \mathrm{NENP} & -48 & 4 \times 10^{-4} & 0.2 & - & {[16,17]} \\ \mathrm{NINO} & -52 & - & 0.3 & - & {[17]} \\ \mathrm{NINAZ} & -100 & - & - & - & \\ \mathrm{AgVP}_{2} \mathrm{~S}_{6} & -400 & - & - & - & {[18]} \\ \mathrm{CuCl}_{3}-\mathrm{BP} & -1.2 & - & - & - & {[19]}\end{array}$




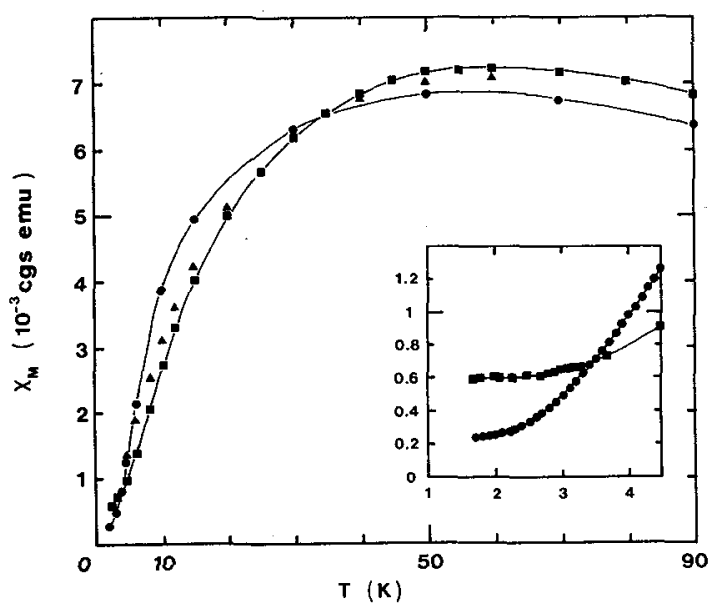

Fig. 3. - Molar susceptibility of NENP along the three crystal axes $a(\bullet), b(\bullet)$ and $c(\Delta)$. The solid lines are guides to the eye.

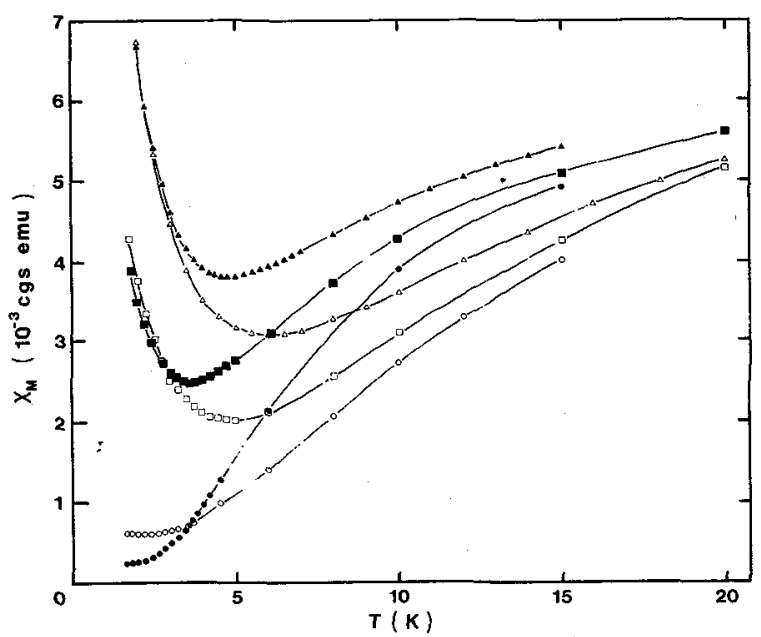

Fig. 4. - Effect of Cu impurities on the molar susceptibility of NENP - pure NENP: $a$ axis (o), $b$ axis ( $)$; NENP: $\mathrm{Cu} 3.6 \times 10^{-3}: a$ axis (a); $b$ axis ( $\left.a\right)$; NENP: $\mathrm{Cu} 6.2 \times 10^{-3}$; $a$ axis $(\Delta) ; b$ axis $(\Delta)$.

is well fitted by a Curie law $\Delta x=C(x) / T$. The experimental Curie constants are about 4.6 times larger than the calculated ones for free $\mathrm{Cu}^{2+}$ impurities. This proves that the ends of finite $\mathrm{Ni}^{2+}$ chains, as for 1D-AF of classical spins, contribute to the susceptibility.

The powder susceptibility of $\mathrm{Ni}\left(\mathrm{C}_{3} \mathrm{H}_{10} \mathrm{~N}_{2}\right)_{2}$ $\mathrm{N}_{3} \mathrm{ClO}_{4}$, alias NINAZ (Fig. 5) and the one of $\mathrm{AgVP}_{2} \mathrm{~S}_{6}$ (Fig. 6) exhibits the extrinsic contribution of defects or impurities at low temperature. Nevertheless, in both compounds, the susceptibility falls in a certain temperature range below the curve calculated by Weng [20] for the gapless $S=1,1 \mathrm{D}-\mathrm{HAF}$. This is a clear manifestation of the Haldane gap. After substracting a suitable extrinsic contribution assumed to be propor-

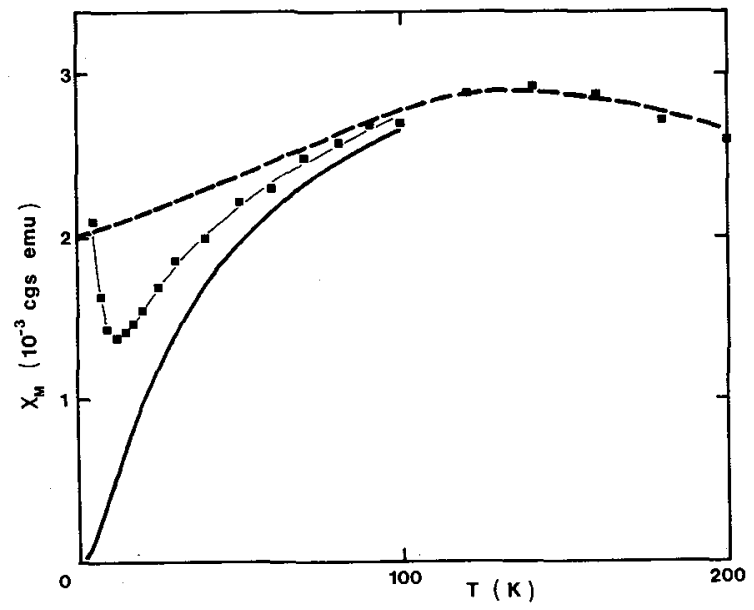

Fig. 5. - Molar magnetic susceptibility of $\mathrm{Ni}\left(\mathrm{C}_{3} \mathrm{H}_{10} \mathrm{~N}_{2}\right)_{2}$ $\mathrm{N}_{3} \mathrm{ClO}_{4}$ (NINAZ) powder. The dashed curve is the calculated susceptibility for gapless $S=1,1 \mathrm{D}-\mathrm{HAF}[20]$. The heavy curve is obtained from experimental data after substraction of a $1 / T$ extrinsic contribution (see text).

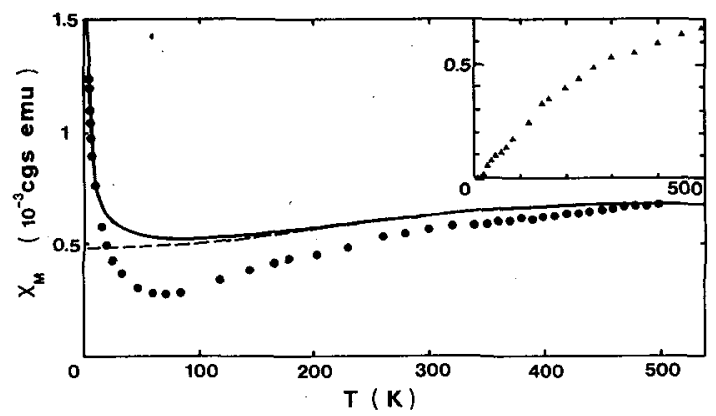

Fig. 6. - Powder susceptibility of $\mathrm{AgVP}_{2} \mathrm{~S}_{6}$. The dashed curve is the calculated susceptibility for the gapless $S=1$, 1D-HAF [20]. The full curve is the calculated one after addition of a suitable $1 / T$ extrinsic contribution. The temperature dependence of the intrinsic observed susceptibility as defined in the text is shown in the inset (from P. Colombet et al. [18]).

tional to $1 / T$, a resulting susceptibility falling down to zero at $T=0$ can be obtained. An estimate of the gap is given by $T_{1 / 2}$ whose value is $32 \mathrm{~K}$ and $160 \mathrm{~K}$ for respectively NINAZ and $\mathrm{AgVP}_{2} \mathrm{~S}_{6}$. This leads to a ratio $E_{\mathrm{G}} /|J|$ of about 0.3 for NINAZ, comparable to the values which can be obtained from susceptibility data in NENP and NINO, and to $E_{\mathrm{G}} /|J| \simeq 0.4$ for $\mathrm{AgVP}_{2} \mathrm{~S}_{6}$.

3.3 MAGNeTIC SPECIFIC heAT. - Standard specific heat measurements have been performed in NENP between 1.2 and $20 \mathrm{~K}$ [21]. In this range, no anomaly indicating an eventual transition to LRO has been detected. the magnetic specific heat $C_{\mathrm{m}}$ was recently 
obtained by J. Ferré et al. [22], from optical linear birefringence measurements. The temperature of the maximum of $C_{\mathrm{m}}$ is fairly consistent with the intrachain exchange determined by other techniques $|J| / k \simeq 50 \mathrm{~K}$. At low temperature, the data can be fitted by $C_{\mathrm{m}}(T)=C_{0}(k T /|J|)^{\alpha}$ with $\alpha=1.4$. This value is much larger than $\alpha=1$ predicted for gapless 1D-HAF but close to the numerical estimate $\alpha=1.6$ for the $S=1,1 \mathrm{D}-\mathrm{HAF}$ [23].

3.4 High FiEld MaGnetization. - In NENP, at temperatures much smaller than the gap, the magnetic moment remains very small up to $H=80 \mathrm{kOe}$ and then exhibits a linear increase at higher fields $H>$ $100 \mathrm{kOe}$ (Fig. 7). This behavior is consistent with the results of numerical calculations on the $S=1,1 \mathrm{D}$ HAF in a field [6]. The critical field which breaks the energy gap is estimated to $H_{\mathrm{c}}=90 \mathrm{kOe}$. The corresponding energy $E_{\mathrm{G}}=g \mu_{\mathrm{B}} H_{\mathrm{c}}$ is about $13 \mathrm{~K}$, for $g=2.2$, which is consistent with the gap value deduced from the susceptibility measurements.

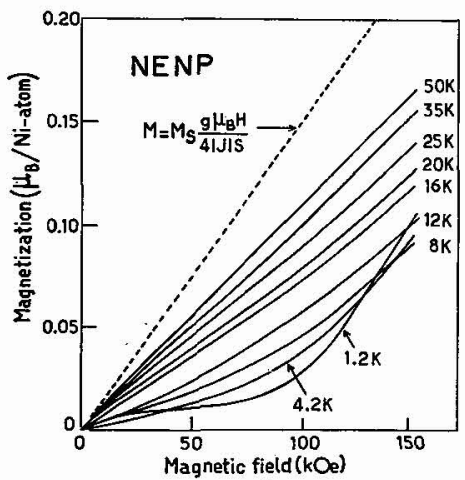

Fig. 7. - Magnetization curves of NENP vs. field and temperature.

3.5 INELASTIC NEUTRON SCATTERING EXPERIMENTS. The opening of a gap in the excitation spectrum can be directly observed by inelastic neutron scattering (INS). To date INS are available only for $\mathrm{Cs} \mathrm{Ni} \mathrm{Cl} 3$ and NENP. In $\mathrm{CsNiCl}_{3}$ a gap at the antiferromagnetic point, $Q=(1 / 3,1 / 3,1)$ has been observed well above $T_{\mathrm{N}}[13,14]$ with a value decreasing from about $0.9 \mathrm{meV}$ (i.e. $E_{\mathrm{G}} /|J| \simeq 0.35$ ) at $T=20 \mathrm{~K}$ to about $0.3 \mathrm{meV}$ just above $T_{N}$ [14]. The observed decrease of the gap when $T$ decreases to $T_{\mathrm{N}}$ could reflect its progressive destruction by 3D fluctuations as discussed in section 2 .

The case of NENP is more favourable because of the absence of LRO. Our INS experiments provided clear observation of Haldane gap [16, 17]. In fact, the experimental data (Fig. 8) revealed two gaps at $q=1$ related to magnetic fluctuations parallel (II) and perpendicular $(\perp)$ to the chain $b$ axis with respective energies $E_{\mathrm{G}}^{\| \prime} \simeq 2.6 \mathrm{meV} \simeq 30 \mathrm{~K}$ and $E_{\mathrm{G}}^{\perp} \simeq 1.2 \mathrm{meV} \simeq 14 \mathrm{~K}$.
These two gaps result from the splitting of the Haldane gap of the pure Heisenberg chain by the planar single ion anisotropy, as shown in figure 1. The average gap value $\bar{E}_{\mathrm{G}}=\left(E_{\mathrm{G}}^{\|}+\mathrm{E}_{\mathrm{G}}^{1}\right) / 2 \simeq 0.43|J|$ which realizes a partial cancellation of the anisotropy effect is close to the predicted value for the $S=1,1 \mathrm{D}$ HAF $(0.41|\mathrm{~J}|)$. Moreover, the observed gap splitting $\left(E_{\mathrm{G}}^{\|}-E_{\mathrm{G}}^{1}\right) / k \simeq 16 \mathrm{~K}$ is in satisfactory agreement with the relation $E_{\mathrm{G}}^{\|}-E_{\mathrm{G}}^{1}=1.6 \mathrm{D}$ coming from numerical calculations [5].

The energy spectrum of the magnetic excitations, for $\hbar \omega_{\mathrm{q}}<6 \mathrm{meV}$, can be fitted to the following dispersion relation.

$$
\hbar \omega_{\mathrm{q}}=2|J|\left[\left(E_{\mathrm{G}}^{\|, \perp} / 2|J|\right)^{2}+(\sin \pi q)^{2}\right]^{1 / 2}
$$

where $q$ is the wave vector expressed in reciprocal lattice units and $|J| / k \simeq 55 \mathrm{~K}$.

Unfortunately the whole excitation spectrum could not be obtained because of the decrease of INS peak intensity with increasing energy and the existence of inelastic modes weakly dispersed in energy around 3.5, 6-7 and 10-11 meV likely due to no non correlated vibrations of protons in ethylenediamine molecules. The effects of temperature and applied magnetic field on the energy modes at $q=1$ [17] are also consistent with the picture of Haldane gap split into two components by magnetic anisotropy. Indeed, when increasing temperature the energy $E_{\mathrm{G}}$ of the lowest mode increases from $0.27|J|$ up to $0.35|J|$ at $T=20 \mathrm{~K}$, reflecting the decrease of anisotropy and thus the tendency of the system to become closer to the pure Heisenberg case.

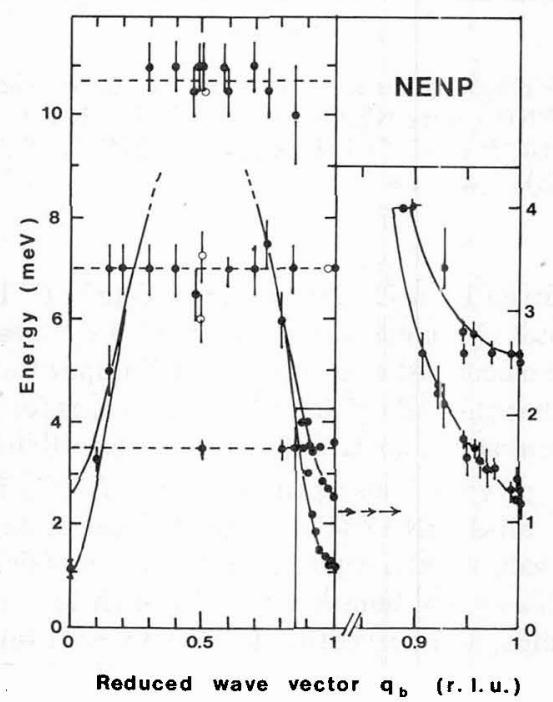

Fig. 8. - Dispersion curves of excitations in NENP below $4.2 \mathrm{~K}$. The solid lines correspond to fits to relation (2) of text. The dashed lines are guides to the eye. 
In applied field along the $c$ axis, the energy of this mode is constant up to the critical field $H_{\mathrm{c}}=90 \mathrm{kOe}$ and then increases with field above $H_{\mathrm{c}}$.

An other important result is the absence of a central peak at low temperature which is proved not only by neutron scattering but also by very recent nuclear magnetic resonance experiments [24]. Indeed, the proton relaxation rate $1 / T_{1}$ decays by more than two orders of magnitude as temperature is reduced below the gap independently of the field orientation, reflecting the progressive vanishing of low energy spin fluctuations.

\section{Conclusion}

The Haldane's prediction of a gap in magnetic excitations of integer spin Heisenberg AF chains has given an impetus to experiments in $S=1$, quasi-1D AF compounds. An increasing number of such systems were recently investigated. They showed the expected low temperature susceptibility decrease and absence of long range order. The results of numerical calculations both for the size of the gap $E_{\mathrm{G}} \simeq 0.4|J|$ and the effect of magnetic anisotropy have been supported by these experiments. An important break-through was realized by the discovery of NENP, a model system fairly one-dimensional which can be obtained in large high quality single crystals. This allowed detailed studies of magnetic excitations by inelastic neutron scattering which well supported the theoretical predictions. The recent development of magnetic resonance experiments in NENP will give a new insight on the spin dynamics and low energy spin fluctuations. An open question remains the correlation length at $T=0$. The valence bond ground state model $[2,4]$ predicts very short range correlations $\xi_{0} \simeq 1$, while numerical calculations [7] give $\xi_{0} \simeq 5$. Neutron scattering data on the $q$ dependence of the integrated intensity in NENP lead to $\xi_{0} \simeq 1.5-2$ [25]. More experimental work is clearly needed on this topic.

\section{Acknowledgments}

We are greatly indebted to our coworkers, J. RossatMignod, W. A. C. Erkelens, W. G. Stirling, C. Vettier, J. Ribas, M. Monfort, R. Vicente and X. Solans. We wish to thank R. Jullien and H. J. Schulz for illuminating discussions, P. Colombet for informing us of his work on $\mathrm{AgVP}_{2} \mathrm{~S}_{6}, \mathrm{~J}$. P. Boucher for communication of his NMR results in NENP, E. Bize and Mrs F. Genet for their help in preparing the manuscript.

[1] Haldane, F. D. M., Phys. Lett. A 93 (1983) 464 and Phys. Rev. Lett. 50 (1983) 1153.

[2] Arovas, D. P., Auerbach, A. and Haldane, F. D. M., Phys. Rev. Lett. 60 (1988) 531;

Haldane, F. D. M. and Rezayi, E. H., Phys. Rev. Lett. 60 (1988) 956.
[3] Ziman, T. and Schulz, H. J., Phys. Rev. Lett. 59 (1987) 140.

[4] Affleck, I., Kennedy, T., Lieb, E. H. and Tasaki, H., Phys. Rev. Lett. 59 (1987) 799.

[5] Botet, R. and Jullien, R., Phys. Rev. B 27 (1983) 613 ;

Botet, R., Jullien, J. and Kolb, M., Phys. Rev. $B 29$ (1983) 5216.

[6] Parkinson, J. B. and Bonner, J. C., Phys. Rev. $B 32$ (1985) 4703.

[7] Moreo, A., Phys. Rev. B 35 (1987) 8562.

[8] Betsuyaku, H., Phys. Rev. B 36 (1987) 799.

[9] Kubo, K. and Takada, S., J. Phys. Soc. Jpn 55 (1986) 438;

Saitoh, K., Takada, S. and Kubo, K., J. Phys. Soc. Jpn 56 (1987) 3755.

[10] Nightingale, M. P. and Blote, H. W. J., Phys. Rev. B 33 (1986) 659.

[11] Takahashi, M., Prog. Thor. Phys. 50 (1973) 1519.

[12] Kosevich, Y. A. and Chubukov, A. V., Sov. Phys. JETP 64 (1986) 654.

[13] Buyers, W. J. L., Morra, R. M., Armstrong, R. L., Hogan, M. J., Gerlach, P. and Hirakawa, K., Phys. Rev. Lett. 56 (1986) 371.

[14] Steiner, M., Kakurai, K., Kjems, J. K., Petitgrand, D. and Pynn, R., J. Appl. Phys. 61 (1987) 3953.

[15] Meyer, A., Gleizes, A., Girerd, J. J., Verdaguer, M. and Kahn, O., Inorg. Chem. 21 (1982) 1729.

[16] Renard, J. P., Verdaguer, M., Regnault, L. P., Erkelens, W. A. C., Rossat-Mignod, J. and Stirling, W. G., Europhys. Lett. 3 (1987) 945.

[17] Renard, J. P., Verdaguer, M., Regnault, L. P., Erkelens, W. A. C., Rossat-Mignod, J., Ribas, J., Stirling, W. G. and Vettier, C., J. Appl. Phys. 63 (1988) 3538.

[18] Colombet, P., Lee, S., Ouvrard, G. and Brec, R., J. Chem. Res. $S$ (1987) 134;

Lee, S., Colombet, P., Ouvrard, G. and Brec, R., Mat. Res. Bull. 21 (1986) 317.

[19] De Groot, H. J. M., De Jongh, L. J., Willett, R. D. and Reedijk, J., J. Appl. Phys. 53 (1982) 8038.

[20] Weng, C. Y. and Ph. D. Thesis, Carnegie Institute of Technology (1968).

[21] Erkelens, W. A. X., Thesis, Leiden University (1987) p. 51.

[22] Ferré, J., Jamet, J. P., Landée, C. P., Reza, K. A. and Renard, J. P., Proc. ICM'88 Conf. (Paris) 1988.

[23] Igarashi, H., J. Phys. Soc. Jpn 54 (1985) 516.

[24] Boucher, J. P., Proc. ICM'88 satellite meeting on nuclear methods in magnetism (Münich) 1988.

[25] Regnault, L. P., Rossat-Mignod, J., Renard, J. P., Verdaguer, M. and Vettier, C., Proc. Int. Conf. on Neutron Scattering (Grenoble) 1988. 